\title{
Serum irisin levels in metabolically healthy versus metabolically unhealthy obesity: A case-control study
}

\author{
Somaye Yosaee ${ }^{1,2}$, Reyhane Basirat ${ }^{3}$, Abdolhamid Hamidi ${ }^{4}$, Alireza Esteghamati ${ }^{5}$, Mahmoud Khodadost ${ }^{6,7}$, \\ Farzad Shidfar $^{8}$, Vida Bitarafan ${ }^{9}$, Kurosh Djafarian*10 (D)
}

\section{Abstract}

Background: Metabolically healthy obese (MHO) individuals appear to be protected or more resistant to the progression of obesityrelated metabolic disorders. Hormonal regulation associated with adipose or muscular tissues such as irisin and leptin may facilitate the healthy metabolic profile of MHO cases. In this case-control study, the differences between serum level of irisin was investigated in metabolically unhealthy obese (MUO) and metabolically healthy obese (MHO) individuals.

Methods: The study participants included obese individuals with metabolic syndrome (MetS) ( $\mathrm{n}=51)$ and 2 control groups that included weight matched cases without MetS $(n=51)$ and normal weight cases without MetS $(n=51)$. Diagnosis of MetS was made based on the Adult Treatment Panel III (ATPIII) criteria. Serum levels of leptin and irisin were determined by enzyme-linked immune-sorbent assay (ELISA) kit. Receiver Operator Characteristic (ROC) curve, multiple linear regression, and one-way ANOVA analysis were used in SPSS 16 software. Significant level was set at 0.05 .

Results: Based on the statistical analysis, serum levels of irisin were $2.91 \pm 1.6,3.14 \pm 1.4$, and 4.47 \pm 3.23 (ng/mL) in MUO, MHO, and nonobese metabolically healthy participants, respectively $(\mathrm{P}=0.001)$. Also, serum levels of leptin were $14.06 \pm 12.4,11.2 \pm 9.3$, and $7.09 \pm 7.1(\mathrm{ng} / \mathrm{mL})$ in MUO, MHO, and nonobese metabolically healthy cases, respectively $(\mathrm{p}=0.002)$. After adjusting for demographic variables, a significant association was found between irisin and study groups $(\beta=0.77, \mathrm{P}=0.001)$, weight $(\beta=-0.03, p=0.014)$, BMI $(\beta=-0.11, p=0.006)$, TG $(\beta=-0.003, p=0.025)$, fat mass $(\beta=-0.04, p=0.046)$, and fat free mass $(\beta=0.08, p=0.014)$.

Conclusion: Obese patients with/without MetS had lower level of irisin than normal weight participants.

Keywords: Irisin, Obesity, Metabolic syndrome, Leptin

Conflicts of Interest: None declared

Funding: Tehran University of Medical Sciences

\section{*This work has been published under CC BY-NC-SA 1.0 license.}

Copyright $\odot$ Iran University of Medical Sciences

Cite this article as: Yosaee S, Basirat R, Hamidi A, Esteghamati A, Khodadost M, Shidfar F, Bitarafan V, Djafarian K. Serum irisin levels in metabolically healthy versus metabolically unhealthy obesity: A case-control study. Med J Islam Repub Iran. 2020 (11 May);34:46. https://doi.org/10.47176/mjiri.34.46

\section{Introduction}

Management of obesity, as the sixth most important risk

Corresponding author: Dr Kurosh Djafarian, kdjafarian@tums.ac.ir

1. Department of Nutrition Sciences, School of Health, Larestan University of Medical Sciences, Larestan, Iran

2. Department of Nutrition, Emam Reza Teaching Hospital, Larestan University of Medical Sciences, Larestan, Iran

3. Nutrition Research Center, Department of Clinical Nutrition, School of Nutrition and Food Sciences, Shiraz University of Medical Sciences, Shiraz, Iran

4. Department of Clinical Nutrition, School of Nutritional Sciences and Dietetics, Tehran University of Medical Sciences, Tehran, Iran

5. Endocrinology and Metabolism Research Center (EMRC), Vali-Asr Hospital, Tehran University of Medical Sciences, Tehran, Iran

6. Department of Epidemiology, Faculty of Health, Shahid Beheshti University of Medical Sciences, Tehran, Iran

7. Department of Epidemiology, Faculty of Health, Iran University of Medical Sciences, Tehran, Iran

8. Department of Nutritional Sciences, School of Public Health, Iran University of Medical Sciences, Tehran, Iran

9. Adelaide Medical School and National Health and Medical Research Council of Australia (NHMRC), Center of Research Excellence in Translating Nutritional Science to Good Health, University of Adelaide, Adelaide, Australia

10. Department of Clinical Nutrition, School of Nutritional Sciences and Dietetics, Tehran University of Medical Sciences, Tehran, Iran factor for global health, is a puzzling issue for nutritionists

\section{$\uparrow$ What is "already known” in this topic:}

Irisin is a novel exercise-induced adipo-myokine, and it has been proposed that this myokine is involved in various procedures such as obesity, glucose hemostasis, and insulin secretion.

\section{$\rightarrow$ What this article adds:}

This study revealed a meaningful difference between the serum levels of irisin in MHO and MUO cases and nonobese metabolically healthy individuals (Nonobese metabolically healthy participants had the highest irisin levels.). Obese patients with/without MetS had higher levels of leptin than normal weight individuals. The elevated irisin level may decrease the risk of MetS development by reducing TG levels. 
(1). Based on the BMI criteria, the aforementioned condition affects more than 600 million people worldwide (2). Obesity is often associated with an increased risk of metabolic syndrome (MetS), type 2 diabetes mellitus (T2DM), beta cell dysfunction, and cardiovascular diseases (CVD) (3). Recently, it has been o bserved that a subset of obese cases appears to be protected or more resistant to the progression of obesity-related metabolic disorders (4-6). These individuals, known as metabolically healthy obese (MHO), are considered by having excessive body fat, while presenting a favorable metabolic profile such as sufficient insulin action, normal levels of liver enzyme function, no sign of dyslipidemia or hypertension as well as high level of cardiorespiratory fitness compared to the metabolically unhealthy obese (MUO) (5-7). The prevalence of the MHO phenotype varies largely depending on ethnicity, age, physical activity, life style, etc. $(8,9)$. Numerous investigations have been conducted to determine the underlying mechanism for the aforementioned protection in MHO individuals. It has been proposed that some cytokines secreted from adipose and muscular tissues play a crucial role in $\mathrm{MHO}$ cases. Leptin and irisin are 2 cytokines that interact with other parameters to facilitate the healthy metabolic profile of MHO (10).

Leptin, as an important anti-obesity adipocytokine, plays a key role in regulation of energy homeostasis, obesity, diabetes mellitus, and other metabolic condition (11). Despite the unclear mechanism of leptin action in metabolic condition, some new investigations demonstrated a relationship between leptin and MHO phenotype. Irisin, as a novel exercise-induced adipo-myokine, is involved in the fat burning and inhibiting weight gain and glucose/lipid metabolism (12). Based on the aforementioned characteristics, irisin may serve as an attractive candidate for prevention and treatment of obesity and its associated problems (12, 13). The present study aimed to investigate the differences in the serum levels of irisin and leptin between MHO, MUO, and nonobese metabolically healthy individuals. Moreover, this study aimed to determine the suitable cutoff and associated sensitivity and specificity of serum irisin to predict metabolic syndrome.

\section{Methods}

\section{Participants and study design}

This matched case-control study was conducted after being approved by the Ethics Committee of TUMS (code No: 26402-93-02-161). The case/control ratio was 1:2. Participants were 51 overweight/obese patients with newly diagnosed MetS (MUO) and 2 control groups age- and gendermatched with MetS patients, including $51 \mathrm{MHO}$ and 51 nonobese metabolically healthy participants. All cases were recruited from the Endocrinology Center of Tehran University of Medical Sciences (TUMS). Based on the body mass index (BMI), non-MetS control participants were classified into 2 groups: lean (BMI 18.5-24.9 kg/m²); overweight/obese $\left(\mathrm{BMI} \geq 25 \mathrm{~kg} / \mathrm{m}^{2}\right)$. All participants received an explanation of the study purpose and signed a written informed consent for participation in the study.

Those with a history of coronary artery disease, acute or chronic renal failure, acute infection within the last week, acute or chronic hepatic failure, uncontrolled thyroid disorder, use of medications for dyslipidemia or hypertension, presence of any chronic inflammatory and autoimmune disease, and any known malignancies were excluded from this study. Other nonpathological exclusion criteria included pregnancy, breast feeding, post menopause, smoking, being a professional athlete, or having a special diet for any reason provided by a qualified dietitian. MetS was diagnosed according to the National Cholesterol Education Program Adult Treatment Panel III criteria (NCEP- ATP III) (14).

\section{Body composition and anthropometrics measurement}

Body composition was measured by bioelectrical impedance analysis (BIA) (model TANITA BC-418), which records body water content, fat mass (FM), and fat free mass (FFM). Height, weight, BMI, and waist circumference (WC) were calculated in all participants. Anthropometric measurements were obtained using standard protocols (15). WC was measured in the midline between the costal margin and the iliac crest of hip, and the aforementioned measuring was done after normal expiration.

\section{Clinical parameters measurement}

All blood samples were obtained from all participants after a 12-hour fasting and immediately centrifuged. Triglycerides (TG) and HDL-C were measured using spectrophotometry kit (Pars Azmun, Iran). Fasting blood sugar (FBS) was measured by a glucose oxidase procedure. Serum levels of leptin and irisin were measured by ELISA kit (Reutlingen / Germany). Also, blood pressure was measured on the nondominant upper extremity in the cases in a sitting position after they rested at least for 10 minutes.

\section{Statistical analysis}

SPSS 16.0 software (SPSS Inc., Chicago, IL, USA) was used for basic statistical analysis. Continuous variables were described as mean \pm SD. One-way analysis of variance was used to assess comparisons between the 3 groups. When the output of the ANOVA statistical test was meaningful, LSD test was done to find which means differed. Also, the analysis of covariance (ANCOVA) was used to adjust body fat components related to estimated irisin level among the various study groups. Receiver Operator Characteristic (ROC) curve was used to determine the suitable cutoff point and related irisin sensitivity and specificity for predicting metabolic syndrome. Multiple linear regression analysis was used to assess the relationships between irisin and other examined parameters. A $p$ value $<0.05$ was considered as statistically significant.

\section{Results}

The characteristics and MetS components of the cases in various groups were presented in Table 1. Blood pressure, FBS, TG, HDL-c, and number of MetS components were not significantly different between the MHO and nonobese metabolically healthy participants $(\mathrm{p}>0.05)$. Compared to participants without MetS (MHO and nonobese metabolically healthy participants), MUO had significantly higher level of WC, BMI, blood pressure, FBS, TG, and number 


\begin{tabular}{|c|c|c|c|c|c|}
\hline \multirow[t]{2}{*}{ Variable } & $\begin{array}{l}\text { MUO } \\
(n=51)\end{array}$ & $\begin{array}{c}\text { MHO } \\
(\mathrm{n}=51)\end{array}$ & $\begin{array}{c}\text { Nonobese metabolically } \\
\text { healthy }(n=51)\end{array}$ & $\begin{array}{c}\text { Total } \\
(\mathrm{n}=153)\end{array}$ & $\mathrm{p}^{*}$ \\
\hline & Mean \pm SD & Mean \pm SD & Mean \pm SD & Mean \pm SD & \\
\hline Age (year) & $37.5 \pm 6.5$ & $36.9 \pm 6.4$ & $35.9 \pm 6.3$ & $36.8 \pm 6.4$ & 0.473 \\
\hline \multirow{2}{*}{$\begin{array}{l}\text { Age (year) } \\
\text { Sex }^{* *}\end{array}$} & $43(84.3)$ & $43(84.3$ & $44(86.3)$ & $130(85)$ & 0.944 \\
\hline & $8(15.7)$ & $8(15.7)$ & $7(13.7)$ & $23(15)$ & \\
\hline Weight(Kg) & $92.5 \pm 13.3^{\mathrm{a}}$ & $89.1 \pm 13.7^{\mathrm{a}}$ & $69.1 \pm 6.8^{b}$ & $83.6 \pm 15.6$ & $<0.001$ \\
\hline Height (cm) & $171.5 \pm 7.7$ & $172.2 \pm 7.0$ & $171.1 \pm 6.8$ & $171.6 \pm 7.1$ & 0.742 \\
\hline $\mathrm{WC}(\mathrm{cm})^{*}$ & $105.1 \pm 8.1^{\mathrm{a}}$ & $100.2 \pm 11.3^{\mathrm{b}}$ & $87.02 \pm 7^{\mathrm{c}}$ & $97.4 \pm 11.8$ & $<0.001$ \\
\hline $\operatorname{BMI}\left(\mathrm{kg} / \mathrm{m}^{2}\right)^{*}$ & $31.4 \pm 3.7^{\mathrm{a}}$ & $29.9 \pm 3.6^{\mathrm{b}}$ & $23.6 \pm 1.4^{\mathrm{c}}$ & $28.3 \pm 4.5$ & $<0.001$ \\
\hline $\mathrm{WHR}^{*}$ & $97 \pm 0.1^{\mathrm{a}}$ & $93 \pm 0.1^{\mathrm{b}}$ & $0.9 \pm 0.1^{\mathrm{c}}$ & $0.93 \pm 0.1$ & $<0.001$ \\
\hline Fat index & $8.6 \pm 2.8^{\mathrm{a}}$ & $7.8 \pm 2.6^{\mathrm{a}}$ & $4.6 \pm 1.7^{\mathrm{b}}$ & $7.0 \pm 2.9$ & $<0.001$ \\
\hline $\mathrm{SBP}^{*}$ & $121.2 \pm 11.7^{\mathrm{a}}$ & $113.8 \pm 8.6^{\mathrm{b}}$ & $111.3 \pm 12.2^{\mathrm{b}}$ & $115.4 \pm 11.6$ & $<0.001$ \\
\hline DBP* & $80.8 \pm 9.1^{\mathrm{a}}$ & $75.7 \pm 6.6^{\mathrm{b}}$ & $74.1 \pm 8.3^{\mathrm{b}}$ & $76.8 \pm 8.5$ & $<0.001$ \\
\hline FBS* & $116.2 \pm 37.3^{\mathrm{a}}$ & $97.8 \pm 20.4^{\mathrm{b}}$ & $95.2 \pm 7.6^{\mathrm{b}}$ & $103.1 \pm 26.4$ & $<0.001$ \\
\hline $\mathrm{TG}^{*}$ & $266.3 \pm 203.6^{a}$ & $131.0 \pm 87.4^{b}$ & $117.5 \pm 67.8^{b}$ & $171.6 \pm 149.0$ & $<0.001$ \\
\hline HDL* & $50.3 \pm 8.3^{\mathrm{a}}$ & $54.3 \pm 5.6^{\mathrm{b}}$ & $55.1 \pm 9.2^{\mathrm{b}}$ & $53.2 \pm 8.1$ & 0.006 \\
\hline MetS* components & $3.3 \pm 0.6^{\mathrm{a}}$ & $1.0 \pm 0.7^{\mathrm{b}}$ & $0.8 \pm 0.6^{\mathrm{b}}$ & $1.7 \pm 1.3$ & $<0.001$ \\
\hline Fat free mass $(\%)$ & $72.9 \pm 6.2^{\mathrm{a}}$ & $74 \pm 6.6^{\mathrm{a}}$ & $80.6 \pm 6^{\mathrm{b}}$ & $75.8 \pm 7.1$ & $<0.001$ \\
\hline Fat mass $(\%)$ & $27.1 \pm 6.2^{\mathrm{a}}$ & $25.9 \pm 6.6^{\mathrm{a}}$ & $19.4 \pm 6.1^{b}$ & $24.1 \pm 7.1$ & $<0.001$ \\
\hline Trunk fat (\%) & $28.9 \pm 5.4^{\mathrm{a}}$ & $27.9 \pm 6.4^{\mathrm{a}}$ & $20.6 \pm 6^{\mathrm{b}}$ & $25.8 \pm 7$ & $<0.001$ \\
\hline Right hand (\%) & $26.1 \pm 8.4^{\mathrm{a}}$ & $25.2 \pm 7.7^{\mathrm{a}}$ & $18.5 \pm 6.2^{\mathrm{b}}$ & $23.3 \pm 8.2$ & 0.001 \\
\hline Left hand $(\%)$ & $26.9 \pm 8.5^{\mathrm{a}}$ & $26.2 \pm 7.9^{\mathrm{a}}$ & $19.1 \pm 6.6^{\mathrm{b}}$ & $24.06 \pm 8.4$ & $<0.001$ \\
\hline Right leg (\%) & $24.2 \pm 7.9^{\mathrm{a}}$ & $22.7 \pm 7.9^{\mathrm{a}}$ & $17.4 \pm 7.2^{\mathrm{b}}$ & $21.4 \pm 8.2$ & $<0.001$ \\
\hline Left leg $(\%)$ & $24.7 \pm 8.9^{\mathrm{a}}$ & $22.9 \pm 7.7^{\mathrm{a}}$ & $17.6 \pm 7^{\mathrm{b}}$ & $21.7 \pm 8.4$ & $<0.001$ \\
\hline Leptin(ng/ml) & $14.0 \pm 12.4^{\mathrm{a}}$ & $11.2 \pm 9.3^{\mathrm{a}}$ & $7.1 \pm 7.1^{\mathrm{b}}$ & $10.8 \pm 10.2$ & 0.002 \\
\hline $\operatorname{Irisin}(\mathrm{ng} / \mathrm{ml})$ & $2.9 \pm 1.6^{\mathrm{a}}$ & $3.1 \pm 1.4^{\mathrm{a}}$ & $4.5 \pm 3.2^{\mathrm{b}}$ & $3.5 \pm 2.3$ & 0.001 \\
\hline
\end{tabular}

Values are analyzed by one-way ANOVA, values are mean \pm SD.

Dissimilar values $(\mathrm{a}, \mathrm{b}, \mathrm{c})$ of each row are significantly different.

** Value are $\mathrm{N}(\%)$

* WC: Waist Circumference, BMI: Body Mass Index, WHR: Waist to Hip Ratio, SBP: Systolic Blood Pressure, DBP: Diastolic Blood Pressure, FBS: Fasting Blood Sugar, TG: Triglyceride, HDL: High Density Lipoprotein, MetS: Metabolic Syndrome.

of MetS components $(\mathrm{p}<0.001)$. MUO patients showed significant lower HDL-c $(\mathrm{p}=0.006)$.

Based on the results of the statistical analysis, leptin was meaningfully different between $\mathrm{MHO}$ and MUO and nonobese metabolically healthy participants (Table 1). Also, nonobese metabolically healthy participants had lower levels of leptin $(7.1 \pm 7.1)$ compared to the obese with/without Mets (MHO and MUO) (11.2 \pm 9.3 and $14.06 \pm 12.4$, respectively) $(\mathrm{p}=0.002)$ (Table 1$)$. Based on ANCOVA results, there is still a considerable relationship between leptin and study groups $(p=0.015)$ (Table 2).

Also, serum irisin level was significantly different between obese participants with/without Mets (MHO and MUO) and nonobese metabolically healthy participants
(Table 1). Nonobese metabolically healthy cases had higher levels of irisin (4.5 \pm 3.23$)$ compared to the obese with/without Mets (MHO and MUO) (3.14 \pm 1.40 and 2.91 \pm 1.60 , respectively) ( $\mathrm{p}=0.001)$ (Table 1). Also, after adjustment for fat mass, there was still a significant association between irisin and study groups (ANCOVA: $\mathrm{p}=0.002$ ), so the mean of irisin in $\mathrm{MUO}$ and $\mathrm{MHO}$ patients was significantly lower compared to average concentrations in the nonobese metabolically healthy cases (Tables 2 and 3 ).

A ROC curve was used to identify the ability of irisin levels to predict the MUO phenotype. Area under the curve (AUC) and ROC curves for the ability of irisin levels to predict MUO phenotype are shown in Figure 1. It can be seen that the cut off for irisin were $5.5(\mathrm{ng} / \mathrm{ml})$, with the

Table 2. ANCOVA for highlighting the association between irisin and leptin with study groups

\begin{tabular}{|c|c|c|c|c|c|c|}
\hline & Source & Sum of squares & $\mathrm{df}$ & Mean square & $\mathrm{F}$ & $\mathrm{p}$ \\
\hline \multirow{3}{*}{ Leptin } & Intercept & 3382.31 & 1 & 3382.31 & 90.43 & $<0.001$ \\
\hline & Fat mass & 8949.26 & 1 & 8949.26 & 239.28 & $<0.001$ \\
\hline & Study groups & 325.48 & 2 & 162.74 & 4.35 & 0.015 \\
\hline \multirow{7}{*}{ Irisin } & Error & 5497.85 & 147 & 37.40 & & \\
\hline & Total & 33446.96 & 151 & & & \\
\hline & Intercept & 81.58 & 1 & 81.58 & 16.23 & $<0.001$ \\
\hline & Fat mass & 3.06 & 1 & 3.06 & 0.61 & 0.437 \\
\hline & Study groups & 67.12 & 2 & 33.56 & 6.68 & 0.002 \\
\hline & Error & 728.64 & 145 & 5.02 & & \\
\hline & Total & 2640.96 & 149 & & & \\
\hline
\end{tabular}

Table 3. Estimated marginal means of irisin and leptin among MUO, MHO, and nonobese metabolically healthy participants

\begin{tabular}{|c|c|c|c|c|}
\hline & $\begin{array}{l}\mathrm{MUO}^{*} \\
(\mathrm{n}=51)\end{array}$ & $\begin{array}{l}\mathrm{MHO}^{*} \\
(\mathrm{n}=51)\end{array}$ & $\begin{array}{c}\text { Nonobese metabolically } \\
\text { healthy }(n=51)\end{array}$ & $\mathrm{p}^{*}$ \\
\hline & $\mathrm{SD} \pm$ mean & $\mathrm{SD} \pm$ mean & $\mathrm{SD} \pm$ mean & \\
\hline Leptin & $10.5 \pm 0.9^{\mathrm{a}}$ & $9.1 \pm 0.7^{\mathrm{a}}$ & $13.0 \pm 0.9^{\mathrm{b}}$ & 0.011 \\
\hline Irisin & $2.8 \pm 0.3^{\mathrm{a}}$ & $3.1 \pm 0.3^{\mathrm{a}}$ & $4.6 \pm 0.3^{b}$ & 0.022 \\
\hline
\end{tabular}

Values are analyzed by ANCOVA, values are mean \pm SE

Dissimilar values $(\mathrm{a}, \mathrm{b}, \mathrm{c})$ of each row are meaningfully different.

*MUO: Metabolic Unhealthy Obese. MHO: Metabolic Healthy Obese 


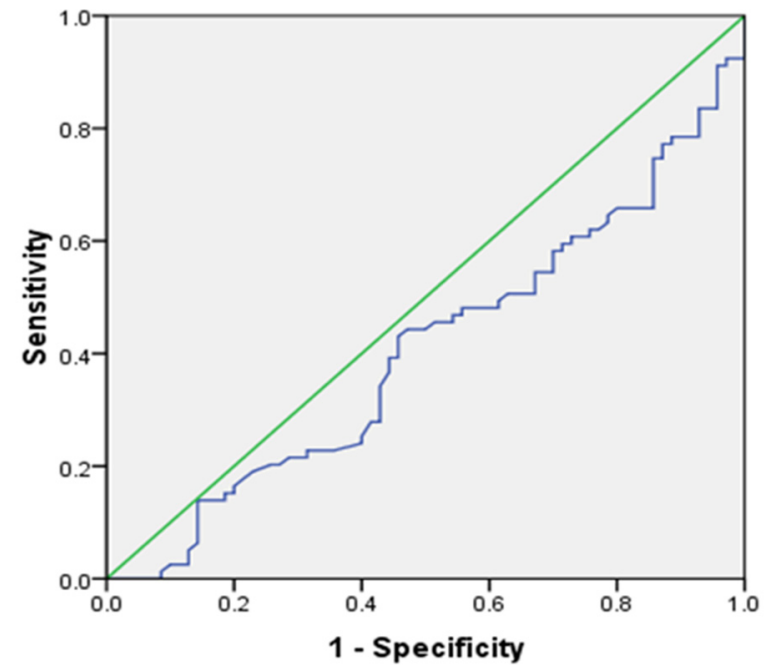

Fig. 1. The ROC curve for determining the irisin cutoff point in diagnosing MetS

area under the Receiver Operator Characteristic curve was 0.40 (95\%CI for AUC: 0.31-049, $\mathrm{p}<0.04)$.

Analysis of linear regression was used to quantifying the possible relation between irisin and other study parameters (Table 4). Based on linear regression analyzing with crude model (model 1), there was a significant association between irisin and study groups $(\beta=0.78, p=0.001)$, weight $(\beta=-0.02, p=0.021)$, BMI $(\beta=-0.11, p<0.001)$, TG $(\beta=-$ $0.003, p=0.013)$ and fat free mass $(\beta=0.06, p=0.012)$. Also, in model 2, with adjusting for demographic variables, there was a considerable meaningful association between irisin and study groups $(\beta=0.77, p=0.001)$, weight $(\beta=-0.03$, $\mathrm{P}=0.014)$, BMI $(\beta=-0.11, p=0.006)$, TG $(\beta=-0.003$, $p=0.025)$, fat mass $(\beta=-0.04, p=0.046)$, and fat free mass $(\beta=0.08, P=0.014)$, fat index $(\beta=-0.15, p=0.034)$. In model 3 , with adjustment for all variables with $p$-value $<0.2$, there was still a significant association between irisin and the study group $(\beta=0.99, p=0.042)$. With increasing one unit in the study group, from MetS to healthy obese group and healthy obese to the normal weight group, the adjusted serum level of irisin was increased by on average $0.99 \mathrm{ng} / \mathrm{mL}$.

In multiple linear regression analysis with backward method (model 4), there was a significant association between irisin and study groups $(\beta=0.75, p=0.001)$.

In study groups, non-obese metabolically healthy subjects group have more serum irisin in compare with $\mathrm{MHO}$ and MUO groups so that there is an increasing trend of irisin level in study groups. In multiple linear regression analysis with backward method, there was a meaningful association between irisin and study groups $(\beta=0.99$, $\mathrm{p}=0.042$ ). In study groups, non-obese metabolically healthy subjects group have more serum irisin in compare with MHO and MUO groups. It means that the MUO group of study meaningfully has a low level of irisin compared to other individuals in other groups (Fig. 2).

Also, there is significant association between irisin and number of MetS components $(\beta=-0.27, p=0.053)$ and study groups $(\beta=0.78, p=0.001)$. With increasing one unit in number of MetS components, the irisin were decreased by 0.27 , so that there is a decreasing trend of irisin according to number of MetS components (Fig. 3).

\section{Discussion}

In the present study, a meaningful difference was found between the serum levels of irisin in MHO and MUO patients and nonobese metabolically healthy participants. The results are in agreement with several studies that evaluated the possible role of irisin in the initiation and progression of obesity and MetS. Some studies reported that serum levels of irisin were decreased in obese adults (16-18). Irisin is one of the crucial secreted myokines and it has been proposed that this myokine is involved in various procedures such as energy balance, glucose hemostasis, insulin secretion, and action $(13,19)$.

Table 4. Multiple linear regression for estimating the relation between irisin and related factors

\begin{tabular}{|c|c|c|c|c|c|c|c|c|}
\hline \multirow[b]{2}{*}{ Variables } & \multicolumn{2}{|c|}{ Model $1^{\mathrm{a}}$} & \multicolumn{2}{|c|}{ Model $2^{b}$} & \multicolumn{2}{|c|}{ Model $3^{c}$} & \multicolumn{2}{|c|}{ Model $4^{\mathrm{d}}$} \\
\hline & $\beta$ & $\mathrm{p}$ & $\beta$ & $\mathrm{p}$ & $\beta$ & $\mathrm{P}$ value & $\beta$ & $\mathrm{p}$ \\
\hline Leptin & -0.01 & 0.471 & -0.02 & 0.292 & - & - & - & - \\
\hline Age (years) & -0.01 & 0.536 & - & - & - & - & - & - \\
\hline Gender & 0.05 & 0.927 & - & - & - & - & - & - \\
\hline Groups $^{\mathrm{e}}$ & 0.78 & $0.001^{*}$ & 0.77 & $0.001^{*}$ & 0.99 & $0.042^{*}$ & 0.75 & $0.001^{*}$ \\
\hline weight & -0.02 & $0.021^{*}$ & -0.03 & $0.014^{*}$ & 0.07 & 0.493 & - & - \\
\hline $\mathrm{WC}^{\mathrm{f}}$ & -0.02 & 0.083 & -0.02 & 0.084 & 0.04 & 0.265 & - & - \\
\hline $\mathrm{BMI}^{\mathrm{f}}$ & -0.11 & $<0.001^{*}$ & -0.11 & $0.006^{*}$ & -0.36 & 0.241 & - & - \\
\hline $\mathrm{SBP}^{\mathrm{f}}$ & 0.001 & 0.962 & 0.002 & 0.925 & - & - & - & - \\
\hline FBS $^{\mathrm{f}}$ & -0.09 & 0.198 & -0.009 & 0.247 & -0.001 & 0.983 & - & - \\
\hline $\mathrm{TG}^{\mathrm{f}}$ & -0.003 & $0.013^{*}$ & -0.003 & $0.025^{*}$ & -0.002 & 0.206 & - & - \\
\hline $\mathrm{HDL}^{\mathrm{f}}$ & 0.001 & 0.954 & 0.001 & 0.962 & - & - & - & - \\
\hline MetS component & -0.27 & 0.053 & -0.26 & 0.073 & 0.35 & 0.178 & - & - \\
\hline Fat mass & -0.04 & 0.055 & -0.04 & $0.046^{*}$ & -0.18 & 0.604 & - & - \\
\hline Fat free mass & 0.06 & $0.012^{*}$ & 0.08 & $0.014^{*}$ & 0.3 & 0.402 & - & - \\
\hline Trunk fat & -0.03 & 0.238 & -0.03 & 0.219 & - & - & - & - \\
\hline Fat index & -0.12 & 0.061 & -0.15 & $0.034^{*}$ & 0.61 & 0.549 & - & - \\
\hline
\end{tabular}

${ }^{\mathrm{b}}$ Adjusted for demographic variables such as sex and age

${ }^{\mathrm{c}}$ Adjusted model (all variables with p-value $<0.2$ entered the model such as sex, groups, weight, WC, BMI, SBP, TG, HDL, MetS component, BMR, fat mass, fat free mass, trunk fat).

${ }^{\mathrm{d}}$ Multivariate backward linear regression model.

e: study groups including; 1: MetS patient, 2: healthy obese, 3: normal weight without MetS

${ }^{\mathrm{f}}$ WC: Waist Circumference, BMI: Body Mass Index, SBP: Systolic Blood Pressure, FBS: Fasting Blood Sugar, TG: Triglyceride, HDL: High Density Lipoprotein *Significant at $\mathrm{p}$ value $<0.05$ 


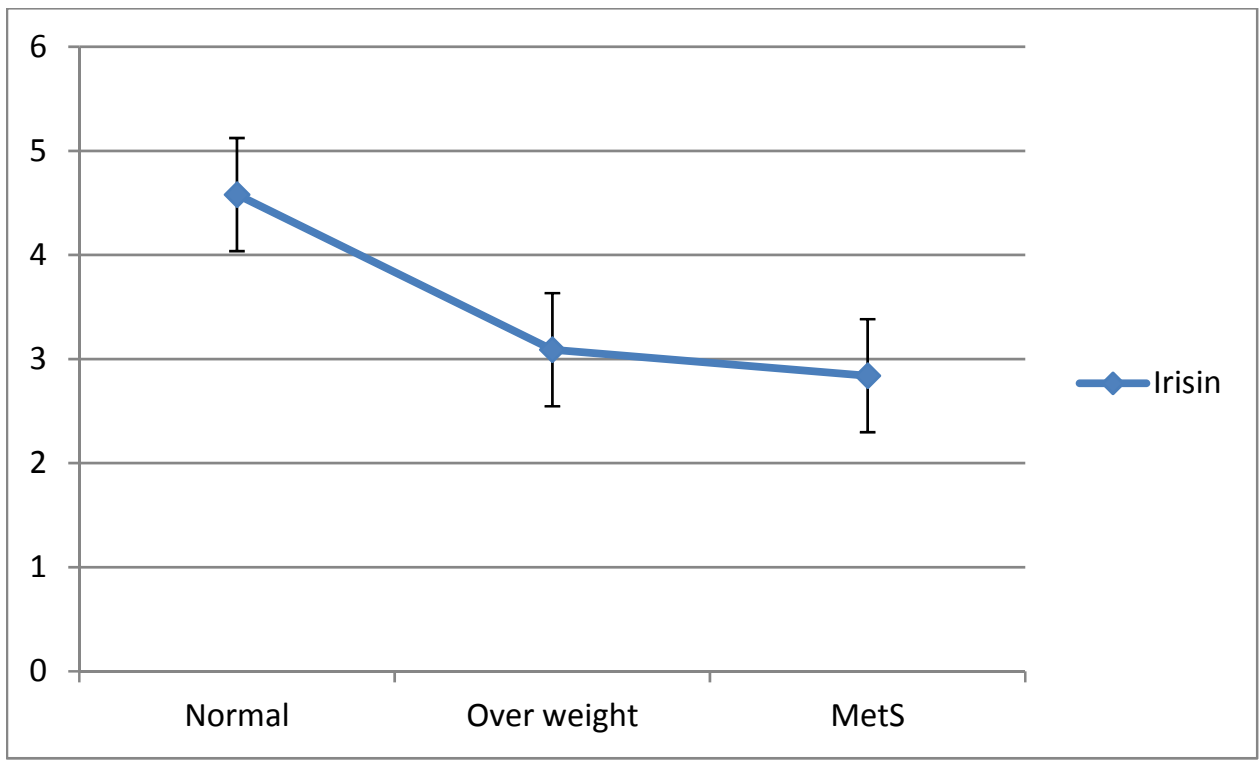

Fig. 2. Trend of irisin (ng/mL) according to study groups

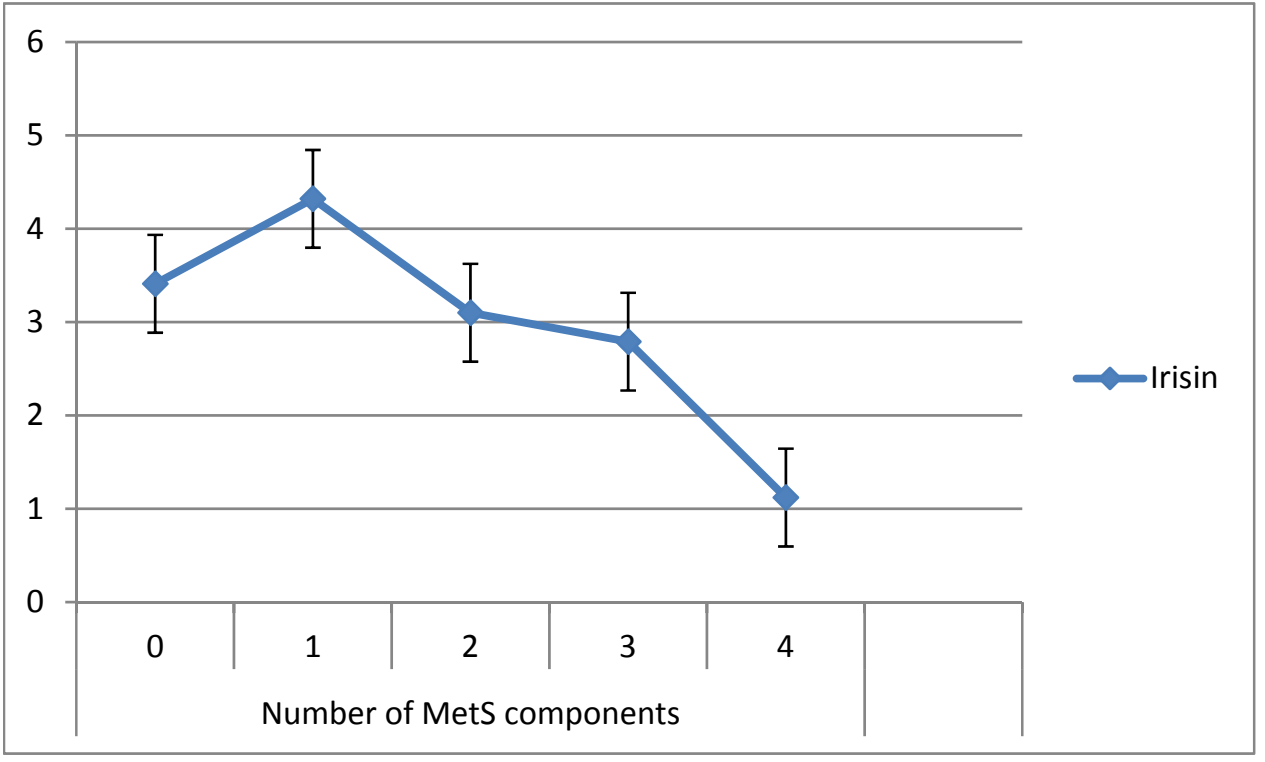

Fig. 3. Trend of irisin (ng/mL) according to number of MetS components

Previous studies reported contradictory results about the possible relationship between irisin levels and MetS. Hee Park et al reported increased serum level of irisin in participants with MetS (20). Furthermore, a positive relationship was found between serum levels of irisin and body weight in some studies (21-24). However, the precise basis for aforementioned discrepancy is unclear (21). However, the present study proposed lower irisin levels in MHO and MUO compared with nonobese metabolically healthy individuals.

The relationship between fat/muscle mass, MetS components, and circulating irisin levels remains a puzzling issue. This study revealed that serum irisin concentrations were negatively correlated with weight, BMI, TG, percent of fatty mass, and number of MetS components in all participants. Also, a positive correlation was found between irisin and percent of fat free mass. Moreover, it seems that high- fat free mass and low-fat mass promote irisin secretion. Our findings are consistent with the results of Moreno-Navarrete and Choi's studies that demonstrated a significant negative correlation between circulating irisin and BMI (13, $25)$ as well as percentage of fatty mass in nondiabetic individuals (13). Another considerable result of this study was the positive correlation of serum irisin with percent of fat free mass. This part of our results was supported by Fukushima Y et al (2016) who reported positive relations between percent lean body mass and serum irisin level (21). However, contradictory effects of body composition (percent of fatty mass/ fat free mass) on serum irisin levels have been reported $(13,24-26)$. Several studies have proposed a positive correlation between serum irisin levels and BMI $(20,27)$ and fatty mass (24).

In accordance with our findings, some researches reflected fat free mass as the main predictor of serum irisin 
(27). Based on some experimental investigations, an increase in muscle mass was accompanied by increased FNDC5 gene expression and $\mathrm{PGC}_{-1 \mathrm{a}}$ and other mitochondrial genes in muscle (28). Activated $\mathrm{PGC}_{1-\alpha}$ and increased expression of FNDC5 gene act as a precursor of irisin and give rise to increasing irisin secretion into blood circulation (21). As previously mentioned, increased circulating irisin was associated with better energy expenditure, fat metabolism, insulin sensitivity, and MetS prevention (13). One of the other crucial consequences of higher irisin level is more browning of white fat performed by increasing uncoupling protein 1 (UCP1) gene expression (29). However, the effect of irisin on lipid profile and glucose concentration remains unclear. Some surveys have reported conflicting results regarding the association between serum level of irisin and lipid/glucose determinants and the issue is open to discussion $(22,24,25,27)$. In the present study, serum irisin concentrations were negatively correlated with TG $(\mathrm{p}<0.05)$. Furthermore, cellular and molecular investigations are needed to determine the possible relations between irisin and TG levels.

In the present survey, no association was found between serum irisin and some clinical parameters such as fasting glucose, BP, and HDL-c. Some studies presented that circulating irisin levels were correlated with FBS (30), BP (20), and HDL-c (31). However, in this study, no correlation was found between serum irisin and HDL-c, FBS, and $\mathrm{BP}$, which is consistent with many previous studies (13, 27).

In agreement with our findings, one study showed that circulating irisin levels were negatively correlated with TG (25). Furthermore, some studies reported that FBS $(25,32)$ and lipid profile (25) were not correlated with irisin levels.

This study had several limitations. First, this was a casecontrol study, so the causal relationship between metabolic and serum levels was not definitive. Another limitation was the small number of females compared to male participants; thus, it was not possible to examine the possible effect of sex. Finally, insulin resistance was not evaluated in the present study. Further study, especially by prospective cohort designs in different populations, are needed to confirm the precise estimation of the causal effect of this study's findings.

\section{Conclusion}

This study reported that the serum level of irisin was associated with TG level negatively and percent of fatty mass, while it was positively correlated with percent of fat free mass. Obese patients with/without MetS had lower irisin level than normal weight participants.

\section{Acknowledgments}

The authors thank all participants who took part in this study. This project was financially supported by Tehran University of Medical Sciences (TUMS).

\section{Conflict of Interests}

The authors declare that they have no competing interests.

\section{References}

1. Lim SS, Vos T, Flaxman AD, Danaei G, Shibuya K, Adair-Rohani H, et al. A comparative risk assessment of burden of disease and injury attributable to 67 risk factors and risk factor clusters in 21 regions, 1990-2010: a systematic analysis for the Global Burden of Disease Study 2010. Lancet. 2012;380(9859):2224-60.

2. Ogden CL, Carroll MD, Fryar CD, Flegal KM. Prevalence of Obesity Among Adults and Youth: United States, 2011-2014. NCHS Data Brief. 2015(219):1-8.

3. Saeedi Borujeni MJ, Esfandiary E, Baradaran A, Valiani A, Ghanadian $\mathrm{M}$, Codoner-Franch $\mathrm{P}$, et al. Molecular aspects of pancreatic beta-cell dysfunction: Oxidative stress, microRNA, and long noncoding RNA. J Cell Physiol. 2019;234(6):8411-25.

4. Primeau V, Coderre L, Karelis AD, Brochu M, Lavoie ME, Messier $\mathrm{V}$, et al. Characterizing the profile of obese patients who are metabolically healthy. Int J Obes. 2011;35(7):971-81.

5. Kim HY, Kim CW, Lee CD, Choi JY, Park CH, Bae SH, et al. Can "healthy" normal alanine aminotransferase levels identify the metabolically obese phenotype? Findings from the Korea national health and nutrition examination survey 2008-2010. Dig Dis Sci. 2014;59(6):1330-7.

6. Lee SH, Han K, Yang HK, Kim MK, Yoon KH, Kwon HS, et al. Identifying subgroups of obesity using the product of triglycerides and glucose: the Korea National Health and Nutrition Examination Survey, 2008-2010. Clin. Endocrinol. 2015;82(2):213-20.

7. Stefan N, Haring HU, Hu FB, Schulze MB. Metabolically healthy obesity: epidemiology, mechanisms, and clinical implications. Lancet Diabetes Endocrinol. 2013;1(2):152-62.

8. Wildman RP, Muntner P, Reynolds K, McGinn AP, Rajpathak S, Wylie-Rosett $\mathrm{J}$, et al. The obese without cardiometabolic risk factor clustering and the normal weight with cardiometabolic risk factor clustering: prevalence and correlates of 2 phenotypes among the US population (NHANES 1999-2004). Arch Intern Med 2008;168(15):1617-24.

9. Phillips CM. Metabolically healthy obesity: definitions, determinants and clinical implications. Rev Endocr Metab Disord. 2013;14(3):21927.

10. Yosaee S, Khodadost M, Esteghamati A, Speakman JR, Shidfar F, Nazari MN, et al. Metabolic Syndrome Patients Have Lower Levels of Adropin When Compared With Healthy Overweight/Obese and Lean Subjects. Am J Mens Health. 2017;11(2):426-34.

11. Saeedi Borujeni MJ, Esfandiary E, Taheripak G, Codoner-Franch P, Alonso-Iglesias E, Mirzaei H. Molecular aspects of diabetes mellitus: Resistin, microRNA, and exosome. J Cell Biochem. 2018;119(2):1257-72.

12. Bostrom P, Wu J, Jedrychowski MP, Korde A, Ye L, Lo JC, et al. A PGC1-alpha-dependent myokine that drives brown-fat-like development of white fat and thermogenesis. Nature. 2012;481(7382):463-8.

13. Moreno-Navarrete JM, Ortega F, Serrano M, Guerra E, Pardo G, Tinahones F, et al. Irisin is expressed and produced by human muscle and adipose tissue in association with obesity and insulin resistance. $\mathrm{J}$ Clin Endocrinol Metab. 2013;98(4):E769-78.

14. Expert Panel on Detection E, Treatment of High Blood Cholesterol in A. Executive Summary of The Third Report of The National Cholesterol Education Program (NCEP) Expert Panel on Detection, Evaluation, And Treatment of High Blood Cholesterol In Adults (Adult Treatment Panel III). Jama. 2001;285(19):2486-97.

15. World Health Organization. Physical status: the use and interpretation of anthropometry. WHO Technical Report Series No. 854. Geneva, Switzerland: WHO, 1995.

16. Fukushima Y, Kurose S, Shinno H, Thi Thu HC, Takao N, Tsutsumi $\mathrm{H}$, et al. Effects of Body Weight Reduction on Serum Irisin and Metabolic Parameters in Obese Subjects. Diabetes Metab. 2016;40(5):386-95.

17. Shi X, Lin M, Liu C, Xiao F, Liu Y, Huang P, et al. Elevated circulating irisin is associated with lower risk of insulin resistance: association and path analyses of obese Chinese adults. BMC Endocr Disord. 2016;16(1):44.

18. Liu BW, Yin FZ, Qi XM, Fan DM, Zhang Y. The Levels of Serum Irisin as a Predictor of Insulin Resistance in Han Chinese Adults with Metabolically Healthy Obesity. Clin Lab. 2017;63(5):881-6.

19. Stanford KI, Middelbeek RJ, Townsend KL, An D, Nygaard EB, Hitchcox KM, et al. Brown adipose tissue regulates glucose homeostasis and insulin sensitivity. J Clin Investig. 2013;123(1):215- 
23.

20. Park KH, Zaichenko L, Brinkoetter M, Thakkar B, Sahin-Efe A, Joung KE, et al. Circulating irisin in relation to insulin resistance and the metabolic syndrome. J Clin Endocrinol Metab. 2013;98(12):4899907.

21. Fukushima Y, Kurose S, Shinno H, Cao Thi Thu H, Tamanoi A, Tsutsumi $\mathrm{H}$, et al. Relationships between serum irisin levels and metabolic parameters in Japanese patients with obesity. Obes Sci Pract. 2016;2(2):203-9.

22. Ebert T, Gebhardt C, Scholz M, Wohland T, Schleinitz D, Fasshauer $\mathrm{M}$, et al. Relationship Between 12 Adipocytokines and Distinct Components of the Metabolic Syndrome. J Clin Endocrinol Metab. 2018;103(3):1015-23.

23. Chen JQ, Huang YY, Gusdon AM, Qu S. Irisin: a new molecular marker and target in metabolic disorder. Lipids Health Dis. 2015;14:2.

24. Stengel A, Hofmann T, Goebel-Stengel M, Elbelt U, Kobelt P, Klapp BF. Circulating levels of irisin in patients with anorexia nervosa and different stages of obesity--correlation with body mass index. Peptides. 2013;39:125-30

25. Choi YK, Kim MK, Bae KH, Seo HA, Jeong JY, Lee WK, et al. Serum irisin levels in new-onset type 2 diabetes. Diabetes Res. Clin. Pract.. 2013;100(1):96-101.

26. Yang $\mathrm{M}$, Chen $\mathrm{P}$, Jin H, Xie X, Gao T, Yang L, et al. Circulating levels of irisin in middle-aged first-degree relatives of type 2 diabetes mellitus - correlation with pancreatic beta-cell function. Diabetol. Metab. Syndr. 2014;6(1):133.

27. Huh JY, Panagiotou G, Mougios V, Brinkoetter M, Vamvini MT, Schneider BE, et al. FNDC5 and irisin in humans: I. Predictors of circulating concentrations in serum and plasma and II. mRNA expression and circulating concentrations in response to weight loss and exercise. Metab. Clin. Exp. 2012;61(12):1725-38.

28. Shan T, Liang X, Bi P, Kuang S. Myostatin knockout drives browning of white adipose tissue through activating the AMPKPGC1alpha-Fndc5 pathway in muscle. FASEB J. 2013;27(5):1981-9.

29. Sanchis-Gomar F, Perez-Quilis C. The p38-PGC-1alpha-irisinbetatrophin axis: Exploring new pathways in insulin resistance. Adipocyte. 2014;3(1):67-8.

30. Xiang L, Xiang G, Yue L, Zhang J, Zhao L. Circulating irisin levels are positively associated with endothelium-dependent vasodilation in newly diagnosed type 2 diabetic patients without clinical angiopathy. Atherosclerosis. 2014;235(2):328-33.

31. Shoukry A, Shalaby SM, El-Arabi Bdeer S, Mahmoud AA, Mousa MM, Khalifa A. Circulating serum irisin levels in obesity and type 2 diabetes mellitus. IUBMB Life. 2016;68(7):544-56.

32. Timmons JA, Baar K, Davidsen PK, Atherton PJ. Is irisin a human exercise gene? Nature. 2012;488(7413):E9-10; discussion E-1. 